\title{
Eco-dialysis: fashion or necessity
}

\author{
Monika Wieliczko ${ }^{1}$ Jacek Zawierucha ${ }^{2} \cdot{\text { Adrian } \text { Covic }^{3} \cdot \text { Tomasz Prystacki }^{4} \cdot \text { Wojciech Marcinkowski }}^{4}$. \\ Jolanta Małyszko' ${ }^{1}$
}

Received: 13 October 2019 / Accepted: 16 January 2020 / Published online: 1 February 2020

(c) The Author(s) 2020

\begin{abstract}
Hemodialysis (HD) is one of the resource hungry medical interventions. A huge volume of water (about $500 \mathrm{~L}$ ) and significant amounts of energy (over $7 \mathrm{~kW}$ ) are used for a hemodialysis session; over a kilogram of waste is produced during this procedure. Thus, HD contributes to global warming while saving patients' lives. In this paper, we showed these crucial points in HD treatment and possible ways (e.g. modifications in dialysate flow rate) to reduce environmental impact maintaining therapy standards.
\end{abstract}

Keywords Hemodialysis $\cdot$ Protection of environment $\cdot$ Medical waste management $\cdot$ Natural resource management

\begin{abstract}
"After nuclear war, humaninduced global warming is the greatest threat to human life on the planet."

Admiral Chris Barrie
\end{abstract}

\section{Introduction}

Hundreds of reports and research published annually confirm that the last few decades have seen major changes in the climate prevailing on Earth. A number of studies point to the major role played by humans in these changes. A study by John Cook, which analyzed 11,944 papers on global warming and climate change, published in peer-reviewed scientific journals reported that only $0.7 \%$ of the analyzed abstracts questioned the human role in this process, and only $0.3 \%$ of the publications considered this role to be uncertain [1]. The vast majority of climatologists, regardless of their assessment on the genesis of changes taking place, do not question the existence of global warming and the role

Jolanta Małyszko

jolmal@poczta.onet.pl

1 Department of Nephrology, Dialysis and Internal Medicine, Medical University of Warsaw, Warsaw, Poland

2 Fresenius Medical Care Polska S.A., Poznan, Poland

3 Grigore T. Popa' University of Medicine, Iasi, Romania

4 Fresenius Nephrocare Polska Sp. Z O.O., Poznan, Poland of greenhouse gas emissions (including carbon dioxide), deforestation and other processes in driving global climate change. The observed changes, resulting from the increase in temperature on the Earth, concern the majority of physical and biological systems. An undeniable fact is the shrinking of the cryosphere- the withdrawal of mountain glaciers or the reduction of the sea ice range in the Arctic. Water supply problems for inhabitants of many countries are growing, and areas threatened with drought are constantly increasing. Higher temperature on Earth exerts influence on phenology, shifting the range of plants and animals (towards poles and on higher heights) and intensification of extreme weather phenomena. Climate changes particularly affect the aquatic environment-higher concentrations of carbon dioxide in the atmosphere change the $\mathrm{pH}$ of water in the oceans, and the warming of freshwaters has a direct impact on its quality [2]. The changes described above, together with the anticipated population growth, already result in a reduction of resources (water, fossil fuels, food), and in the future may lead to their deficit or total depletion, directly affecting the health and quality of life of the human population. In the human history, the massive climate changes were observed a couple of times. Medieval warming and little ice age are well described by scientists. There are a lot of hypotheses concerning the reasons of these anomalies-some of them indicate the sun activity change, abnormal volcanic activity, and the influence of human activity—deforestation of the land in North America and epidemic diseases [3, 4]. Both Medieval warning and little ice age affected huge areas 
but cannot be treated as global changes. Currently observed climate changes are global-the warming is observed worldwide [5].

Dialysis is one of the most "resource-intensive" fields of medicine. Over $500 \mathrm{~L}$ of water, $7 \mathrm{~kW}$ of energy and more than a kilogram of medical waste are consumed during hemodialysis. The aim of this paper is to analyze the impact of dialysis on the environment and to review possibilities to reduce the use of resources necessary for the implementation of treatments.

\section{Dialysis in the world}

In 2010, 2,618,000 patients were treated worldwide, but the actual number of patients requiring this type of therapy ranged from 4,900,000 according to a conservative model to $9,700,000$ patients in the high-estimated model. Lack of access to treatment in less developed countries (some countries in Asia and Africa) means that over 2,000,000 people do not receive any treatment. It is also assumed that the number of patients undergoing dialysis will double by 2030 around 5,000,000 patients [6]. At the same time, it is assumed that the number of patients with end-stage kidney disease increases by about $6 \%$ per year. Nearly $90 \%$ of patients treated with renal replacement therapy undergo extracorporeal blood purification (hemodialysis, hemodiafiltration or their variants) and only about $10-11 \%$ have peritoneal dialysis [7]. This percentage is different in different countries-in the United States, the percentage of new patients included in the peritoneal dialysis treatment is about 9-10\%, and in Poland or Romania below 5\% [8, 9].

The above data indicate that around 600 million hemodialysis procedures are performed annually around the world. Such a large and growing number of treatments year after year make hemodialysis a serious burden on the natural environment. The most important goal of manufacturers of equipment and medical devices should be the development of technologies that allow for the most economical use of resources. On the other hand, providers should be aware of the impact of treatment on the environment and choose solutions that minimize the negative impact on the environment [10].

\section{Resources used in hemodialysis}

\section{Water}

Hemodialysis is a renal replacement therapy technique based on the use of diffusion (or convection and diffusion in the case of hemodiafiltration). The passage of particles from the blood into the dialysate requires a constant flow of appropriately selected dialysis fluid. The average water consumption for one HD treatment can be even about $500 \mathrm{dm}^{3}$. This means that during 1-year treatment for one patient, the hemodialysis therapy requires about $78 \mathrm{~m}^{3}$ of water. $270,000,000 \mathrm{~m}^{3}$ of water is thus necessary for the treatment of all dialysis patients, which is equal to the annual resource for many poor water countries. Some of the water is irretrievably lost in the technological processes of water treatment. Production of $1 \mathrm{~L}$ of ultrapure water for dialysis requires 1.5-1.7 raw water. It means that about $60-70 \%$ water (depending on water treatment system) is rejected. What is important-the rejected water is still potable and can be used for different, regular purposes (e.g. for windows and floor cleaning, car washing, dish washing, garden watering). Salvage of the rejected water can be directly translated into cost savings. Every year at least $100,000,000 \mathrm{~m}^{3}$ can be saved if we re-use the water rejected by water treatment units only.

The next important and easy to address items are control of the flow of the dialysate and of the use water during priming and rinsing.

\section{Effect of reduced dialysis fluid flow in hemodialysis on water preservation}

Hemodialysis (HD) — a standard of care for patients with end-stage renal disease-is a treatment that removes uremic toxins and excess water from the body and regulates the concentration of electrolytes in plasma. In conventional hemodialysis, the blood is brought into contact with dialysis fluid through membranes in a dialyser. The delivered dose of dialysis is an important predictor of patient outcome $[11,12]$. The National Kidney Foundation's hemodialysis guidelines (DOQI) recommended a target single pool Kt/V $(\mathrm{spKt} / \mathrm{V})$ of 1.4 per hemodialysis session for patients treated thrice weekly, with a minimum Kt/V of 1.2 , in the absence of residual renal function [13]. Dialysis clearance depends on blood and dialysate flow rates, ultrafiltration flow, dialyser membrane surface, mass transfer coefficient for the solute substances and dialysis time [11]. Since the 1960s, the dialysate flow rate $(\mathrm{Qd})$ has routinely been maintained at $500 \mathrm{~mL} / \mathrm{min}$. Thus, HD needs huge amounts of water for dialysate production. In the previous publications, Agar et al. had reported that a 4-h HD with Qd $500 \mathrm{~mL} / \mathrm{min}$ has a water consumption of about $408 \mathrm{~L}$ per individual session treatment, with additional $34 \mathrm{~L}$ and $51 \mathrm{~L}$ being consumed for a 20-min priming phase and 30-min rinsing phase, respectively. A total water consumption for a single hemodialysis session is nearly $500 \mathrm{~L}$. For worldwide HD population, daily water consumption is around 500 million $\mathrm{L}[14,15]$. Hemodiafiltration (HDF) additionally uses the phenomenon of convective transport. The original forms of HDF-classical and on-line HDF-need external infusion of controlled 
amounts of substitution fluid. Classical HDF uses external substitution fluid provided as a sterile solution in plastic bags; HDF on-line (more efficient mode of HDF) prepares the substitution fluid that must be sterile and non-pyrogenic, continuously during the treatment. HDF improves the removal of middle-to-large uremic toxins and since 2012, several randomized trials have compared hemodiafiltration to either low-flux and high-flux HD [16-19]. One of these trials (ESHOL Study) has shown significantly reduced allcause and cardiovascular mortality with hemodiafiltration online compared to high-flux HD [18]. Now many countries in Europe use these treatment methods for their patients (70\% and $50 \%$ of HD sessions in Germany and France, respectively). Based on recent controlled trials, high volume substitution is preferred-the minimum threshold convective dose to ensure patient benefits starts at $23 \mathrm{~L} / 1.73 \mathrm{~m}^{2}$ per session in post-dilution HDF mode [20]. The volume of the infusion solution in classical HDF is limited and 8-10 L of fluid is most commonly used-hence, on-line HDF mode is preferred. When on-line prepared fluid is used, convection also reduces the dialysis fluid flow and this fluid serves as the source of substitution fluid. The same randomized studies used higher fluid flow than in standard hemodialysis Qd $(553 \mathrm{~mL} / \mathrm{min}$ in ESHOL Study, $500-600 \mathrm{~mL} / \mathrm{min}$ in FRENCHIE Study). This means an additional consumption of $70 \mathrm{~L}$ of water per week, $910 \mathrm{~L}$ per month and 10,920 L per year in on-line HDF mode.

Recent studies have shown that for dialysers with features that promote good dialysate distribution, increasing $\mathrm{Qd}$ during standard hemodialysis offers only a modest impact on dialyser performance; conversely, the effects of reducing dialysate flow rate below conventional $500 \mathrm{~mL} / \mathrm{min}$ have not been studied extensively [21]. In trials published between 1984 and 2015 (11 studies), scientists compared the impact of different Qd (500 vs $300 \mathrm{~mL} / \mathrm{min}, 350,500$ and $800 \mathrm{~mL} /$ min [22], 500 vs $400 \mathrm{~mL} / \mathrm{min}$ [19], 400, 500 and $700 \mathrm{~mL} /$ $\min [2,23])$ on diffusion mass transfer of uremic toxins during clinical hemodialysis. In all studies, the mean weight of patients was similar, less than $70 \mathrm{~kg}$. Six studies investigated the influence of flow changes on Kt/V [9, 20]: five studies showed no significant difference between lower vs higher Qd $(400 \mathrm{~mL} / \mathrm{min}$ vs $500 \mathrm{~mL} / \mathrm{min}$ or $500 \mathrm{~mL} / \mathrm{min}$ vs $700 \mathrm{~mL} /$ min); only one study (from Spain) reported a $4 \%$ statistically significant increase of Kt between Qd 400 and $500 \mathrm{~mL} / \mathrm{min}$ and a $2.9 \%$ increase from 500 to $700 \mathrm{~mL} / \mathrm{min}[12,24,25]$. Two studies focused on clearances for urea and creatinine $[24,25]$ and showed that small solute clearance is highly dependent on dialysate flow rate but only when the dialysate flow rate is much less than $200 \mathrm{~mL} / \mathrm{min}$. Four different trials demonstrated that beta-2 microglobulin (beta2MG) clearance was independent of Qd [21, 26, 27]. In three studies, scientists did not find significant differences in phosphorus removal between dialysate flow rates of 400 and $500 \mathrm{~mL} /$ min $[18,20]$. In conclusion, most of the studies did not show the impact of Qd changes on uremic toxins clearance and patients' outcome. Reduction of Qd from 500 to $400 \mathrm{~mL} / \mathrm{min}$ could save approximately $24 \mathrm{~L}$ of water during 4 -h standard hemodialysis for one patient, especially in patients with a body mass less than $70 \mathrm{~kg}$. Hence, it might result in saving 72,312 and $3744 \mathrm{~L}$ of water a week, a month and a year, respectively. On a global ecological scale, it would mean conserving 24 billions of liters of water daily. We need more studies in different populations (e.g. in obese patients) and studies into the effectiveness of HDF taking into account different Qd to be sure that water saving during hemodialysis/hemodiafiltration does not affect the quality of the therapy [28, 29]. We currently do not have enough data to assume that Qd reduction is safe and effective for hemodialysis patients. We do not have the same data to recommend this type of guideline changes although we produce a lot of wastewater using more ultrapure water and the problem of water scarcity is particularly important in areas affected by chronic drought.

Finally, the data presented above are only related to dialysate flow. Modifying priming and rinsing strategies should be carefully considered as a possibility of significant savings or waste of valuable resources.

\section{Electricity}

Hemodialysis is a power-hungry medical intervention. The massive amount of power is used by water, the treatment unit and by HD machines. Hot disinfection of the loop as well as hot disinfection of the dialysis machines are the most energy-consuming processes during renal replacement treatment. It is obvious that proper disinfection is needed to avoid the risk of infections, but we should (re)consider which kind of disinfection is effective and rational from an economical point of view. The modern hemodialysis machines are prepared for providing thermochemical disinfection $\left(84-85^{\circ} \mathrm{C}\right)$. An unsolved issue remains the effectiveness of the distribution loop for hot disinfection. We did not find any studies which proved the advantages of this method. The results of the study NCT01138280-Heat Disinfection of HD Water Treatment System in Hemodialysis Patients has not been posted or published [30]. If we take into consideration that heating of $1 \mathrm{~L}$ of water by $1{ }^{\circ} \mathrm{C}$ consumes $1.16 \mathrm{Wh}$, we should reconsider the sense of such non-economical processes. On the other hand, perhaps using alternative energy sources such as solar panels, would make it possible to reduce the use of highly polluting energy sources.

\section{Medical waste}

The problem of waste produced during medical interventions exists in all countries with developed healthcare 
system. Although many countries introduced legal regulations concerning medical waste management, the problem of the rising quantity of medical waste is growing according to the number of medical procedures provided. Dialysis is one of the medical interventions which produce relatively high amounts of waste. We can estimate that during every HD session even more than $2 \mathrm{~kg}$ of potentially infective waste is produced. It means that every year HD providers produce more than 1 billion $\mathrm{kg}$ waste. It causes serious economical impact - the cost of $1 \mathrm{~kg}$ waste in Europe is about two EUR [31]. Proper selection of waste and using lighter disposables can be directly translated into the cost savings. Complete emptying of the dialysis set plays a key role in reducing the weight of the dialysis waste. Unpublished data from the Fresenius 6008 machine observational study showed that the weight of medical waste can be $150 \mathrm{~g}$ lighter in case of a dialysis session provided with the Fresenius 6008 machine in contrast to the Fresenius 5008S or the Fresenius 4008S machines. Using modern HD machines that allow emptying of the dialysis set helps to save about $24 \mathrm{~kg}$ of medical waste per patient yearly.

\section{Conclusion}

Environment is now an intricate part of our human life. We are responsible to keep it as much as possible in an unchanged shape for the next generations. Even small changes made in our everyday activities could have a big impact on the ecosystem of our planet. The medical services are one important part of daily human activities that massively influence the current waste burden. Renal replacement therapies are one of the most resource-consuming medical technology. To provide a dialysis session we need large amount of water and electricity. Additionally, every HD session produces a significant number of medical wastes. In addition to the massive effect on the environment, resources needed for HD treatment generate serious costs for the healthcare system. The spending for medical waste utilization and its impact on the environment are undisputed. Every year 1 billion kilograms of medical waste produced during dialysis worldwide must be separated, stored, and burnt. Observance of the recommendations below can help to reduce the impact of HD treatment on the environment and will allow to make significant savings.

Next to the nephrologists and their change of thinking, the industry should increase their efforts in the development of more "green" products-more effective water treatments units, less electricity consuming HD machines, light disposables constructed with less harming components (e.g. phthalanes). The future of our planet is in our hands-even small step can cause the huge improvement.
1. Be aware about the impact of your decision on environment.

2. Segregate the waste. Separate the recyclable and medical wastes.

3. Use lightweight dialysers and other disposables.

4. Empty completely the dialysis set-dialyser and bloodlines. Use HD machines equipped with automatic function of emptying the dialysis sets.

5. Carefully consider the dialysis fluid flow and use priming with dialysis fluid instead of saline.

6. Check the energy consumption of your HD machines. If you plan to replace HD machines, choose models consuming less electricity.

7. Use certified, environment-friendly disposables-without phthalanes and other toxic plasticizers.

8. Carefully plan patients' travel routes-you will save time, money and decrease $\mathrm{CO}_{2}$ emission.

9. Use proper function of $\mathrm{HD}$ machine to limit water and electricity consumption.

10. Urgent research efforts to study dialysate regeneration, and waste disposal, by new methods should also be considered.

Open Access This article is licensed under a Creative Commons Attribution 4.0 International License, which permits use, sharing, adaptation, distribution and reproduction in any medium or format, as long as you give appropriate credit to the original author(s) and the source, provide a link to the Creative Commons licence, and indicate if changes were made. The images or other third party material in this article are included in the article's Creative Commons licence, unless indicated otherwise in a credit line to the material. If material is not included in the article's Creative Commons licence and your intended use is not permitted by statutory regulation or exceeds the permitted use, you will need to obtain permission directly from the copyright holder. To view a copy of this licence, visit http://creativecommons.org/licenses/by/4.0/.

\section{References}

1. Cook J, Nuccitelli D, Green S et al (2013) Quantifying the consensus on anthropogenic global warming in the scientific literature. Environ Res Lett 8:24024-24027. https://doi. org/10.1088/1748-9326/8/2/024024

2. Kundzewicz ZW (2008) Konsekwencje globalnych zmian klimatu. Nauka 1:103-118

3. Schurer AP, Hegerl GC, Mann ME, Tett SFB, Phipps SJ (2013) Separating forced from chaotic climate variability over the past millennium. J Clim 26:6954-6973. https://doi.org/10.1175/ JCLI-D-12-00826.1

4. Koch A, Brierley Ch, Maslin MM, Lewis SL (2019) Earth system impacts of the European arrival and great dying in the Americas after 1492. Quat Sci Rev 207:13-36. https://doi. org/10.1016/j.quascirev.2018.12.004

5. Intergovernmental Panel on Climate Change (IPCC). Climate change and land. https://www.ipcc.ch/site/assets/uploa ds/2019/08/4.-SPM_Approved_Microsite_FINAL.pdf. Accessed 8 Dec 2019 
6. Liyanage T, Ninomiya T, Jha V, Neal B, Patrice HM, Okpechi I (2015) Worldwide access to treatment for end-stage kidney disease: a systematic review. Lancet 385(9981):1975-1982

7. Jain AK, Blake P, Cordy P, Garg AX (2012) Global trends in rates of peritoneal dialysis. J Am Soc Nephrol 23(3):533-544

8. USRDS (2018) Annual data report. Reference table D1: percentages and counts of reported ESRD patients by treatment modality

9. Dębska-Ślizień A, Rutkowski B, Rutkowski P, Korejwo G, Gellert R (2018) Stan terapii nerkozastępczej w Polsce 2016. Nefrol Dial Pol 22(1):1-8

10. Żurek G (2008) Zasoby wodne Polski-obecne i przyszłe wyzwania dla praktyki rolniczej. Problemy Inżynierii Rolniczej 16(2):33-40

11. Ward RA, Ouseph R (2001) Increasing dialysate flow rate increase dialyser urea mass transfer-area coefficient during clinical use. Am J Kidney Dis 37(2):316-320

12. Alayoud A, Benyahia M, Montassir D et al (2012) A model to predict optimal dialysate flow. Ther Apher Dial 16(2):152-158

13. National Kidney Foundation (2015) KDOQI clinical practice guideline for hemodialysis adequacy: 2015 update. Am J Kidney Dis 66(5):884-930. https://doi.org/10.1053/j.ajkd.2015.07.015

14. Agar JWM (2015) Green dialysis: the environmental challenges ahead. Semin Dial 28(2):186-192

15. Agar JWM, Simmonds RE, Knight R, Somerville CA (2009) Using water wisely: new, affordable, and essential water conservation practices for facility and home hemodialysis. Hemodial Int 13(1):32-37

16. Grooteman MPC, van den Dorpel MA, Bots ML et al (2012) Effect of online hemodiafiltration on all-cause mortality and cardiovascular outcomes. J Am Soc Nephrol 23(6):1087-1096

17. Ok E, Asci G, Toz M et al (2013) Mortality and cardiovascular events in online haemofiltration (OL-HDF) compared with highflux dialysis: results from the Turkish OL-HDF Study. Nephrol Dial Transpl 28(1):192-202

18. Maduell F, Moreso F, Pons M, ESHOL Study group et al (2013) High-efficiency postdilution online hemodiafiltration reduces all-cause mortality in hemodialysis patients. J Am Soc Nephrol 24(3):487-497

19. Morena M, Jaussent A, Chalabi L et al (2017) Treatment tolerance and patient-reported outcomes favor online hemofiltration compared to high-flux hemodialysis in the erderly. Kidney Int 91(6):1495-1509

20. Canaud B, Köhler K, Sichart JM, Möller S (2019) Global prevalent use, trends and practice in hemodiafiltration. Nephrol Dial Transpl 14(5):1-10
21. Bhimani JP, Ouseph R, Ward R (2010) Effect of increasing dialysate flow rate on diffusive mass transfer of urea, phosphate and $\beta_{2}$-microglobulin during clinical hemodialysis. Nephrol Dial Transpl 25(12):3990-3995

22. Kashiwagi T, Sato K, Kawakami S et al (2013) Effects of reduced dialysis fluid flow in hemodialysis. J Nippon Med Sch 80(2):119-130

23. Guzman G, Yunez A, Molano A, Wancjer BBJ (2018) Effect of dialysate flow on interdialytic weight gain in chronic hemodialysis. Nephrol Dial Transpl 33(suppl 1):i514-i515

24. Albalate M, Perez-Garcia R, de Sequera P et al (2015) Is it useful to increase dialysate flow rate to improve the delivered $\mathrm{Kt}$ ? BMC Nephrol 16(1):20-25

25. Leypoldt JK, Kamerath CD, Gilson JF, Friederichs G (2006) Dialyser clearances and mass transfer-area coefficients for small solutes at low dialysate flow rates. ASAIO J 52(4):404-409

26. Yamamoto K, Matsukawa H, Yakushiji T et al (2007) Technical evaluation of dialysate flow in a newly designed dialyser. ASAIO J 53(1):36-40

27. Hauk M, Kuhlmann MK, Riegel W, Ko H (2000) In vivo effects od dialysate flow rate on $\mathrm{Kt} / \mathrm{V}$ In maintenance hemodialysis patients. Am J Kidney Dis 35(1):105-111

28. Lee WC, Uchino S, Fealy $N$ et al (2003) $\beta_{2}$-Microglobulin clearance with super high-flux hemodialysis: an ex vivo study. Int $\mathbf{J}$ Artif Organs 26(8):723-727

29. Molano-Triviňo A, Wancjer B, Nerio MM, Karopadi AN, Rosner M, Ronco C (2018) Blue planet dialysis: novel water-sparing strategies for reducing dialysate flow. IJAO 41(1):3-10

30. NCT01138280 heat disinfection of HD water treatment system in hemodialysis patients. https://clinicaltrials.gov/ct2/show/results/ NCT01138280. Accessed 16 Sept 2019

31. Picolli G, Nazha M, Ferraresi M, Neve Vigotti F, Pereno A, Barbera S (2015) Eco-dialysis: the financial and ecological costs of dialysis waste products: is a "cradle to cradle" model feasible for planet-friendly haemodialysis waste management. Nephrol Dial Transpl 30(6):1018-1027

Publisher's Note Springer Nature remains neutral with regard to jurisdictional claims in published maps and institutional affiliations. 